\title{
Antecedentes de políticas y organizaciones sociales orientadas a la promoción de la responsabilidad social empresarial en el siglo $X X$
}

\section{History of political and social organizations aimed at promoting corporate social responsibility in the twentieth century}

\author{
Ana Cecilia Chumaceiro H, ${ }^{1}$ Ph.D., Enrique José Chirinos Noroño, ${ }^{2}$ Tec., \\ Ivana Valeria Reyes $\mathrm{H}^{2,3} \mathrm{MC}$.
}

\begin{abstract}
${ }^{1}$ Corporación Universitaria Antonio José de Sucre (CORPOSUCRE). E-mail: anachuma@gmail.com. 2Universidad Nacional Experimental "Rafael María Baralt" (UNERMB). Centro de Estudios e Investigaciones Socioeconómicas y Políticas (CEISEP). E-mail: chirinos.enrique@hotmail.com. ${ }^{3}$ Universidad del Zulia (LUZ). E-mail: valeriark@hotmail.com
\end{abstract}

\section{Resumen.}

El presente artículo identifica los esfuerzos iniciales realizados en el desarrollo de políticas públicas (PP) de responsabilidad social empresarial (RSE) y los instrumentos que existen para su promoción desde el ámbito de Organizaciones a nivel internacional. En este sentido se revisaron los contextos y gestión de: 1) la Comisión Europea, Organización Internacional del Trabajo, Organización para la Cooperación y el Desarrollo Económico, entre otras; 2) las organizaciones internacionales que incentivan la transparencia de la RSE, tal es el caso de la Comisión Europea y Global Reporting Initiative; y, por último, (3) las organizaciones internacionales que impulsan el escrutinio de la RSE, incluidas la Comisión Europea y Norma SA8000. El objetivo de la presente disertación es reflejar los inicios de las políticas orientadas hacia la RSE, abordar la promoción de la RSE por parte de los organismos internacionales haciendo referencia a las principales iniciativas surgidas y que en su mayoría se encuentran en el continente europeo. El método consiste en una revisión bibliográfica, documental y síntesis analítica. A modo de conclusión, el Pacto Global de Naciones Unidas une criterios de comportamiento para las empresas, tanto en los impactos directos en los ciudadanos y en ámbitos de impactos indirectos de efectos como la competencia desleal, la extorsión y el soborno.

Palabras clave: Organizaciones internacionales, promoción de responsabilidad social, empresas, sociedad. 


\section{Abstract.}

This article identifies the initial efforts in developing public policies (PP) Corporate Social Responsibility (CSR) and the tools that exist to promote it from the realm of international organizations. In this regard the contexts and management will be reviewed: 1) the European Commission, International Labour Organization, Organisation for Economic Co-operation and Development, among others; 2) international organizations that encourage transparency of CSR, as in the case of the European Commission and Global Reporting Initiative; and finally, (3) international organizations that promote the scrutiny of CSR, including the European Commission and SA8000. The objective of this dissertation is to reflect the beginnings of policies to CSR, addressing the promotion of CSR by international organizations referring to the major initiatives emerged and most are in Europe. The method consists of a literature review, documentary and analytical synthesis. In conclusion, the United Nations Global Compact unites performance criteria for companies in both the direct impact on citizens and in areas of indirect impacts of effects such as unfair competition, extortion and bribery.

Key words: International Organizations, Promoting Social Responsibility, Business, Society.

\section{Introducción}

Durante el inicio del siglo XXI la promulgación y difusión del Libro verde de la Unión Europea fue uno de los más grandes pasos para la puesta en práctica de RSE (Comisión Europea, 2001), lo cual hace de Europa el líder en los procesos de RSE en el mundo. La Unión de Confederaciones de la Industria y de los Empresarios de Europa, destaca que para sus empresas el beneficio económico no constituye el principal objetivo, lo que permite incluir acciones de RSE en sus planes de desarrollo organizacional, todo ello acorde con las políticas comunitarias y las obligaciones internacionales, incluyendo el cumplimiento de directrices de las Naciones Unidas, la Organización para la Cooperación y el Desarrollo Económicos (OCDE) y la Organización Internacional del Trabajo (OIT), entre las otras, mismas que comienza a impulsar la RSE a partir del año 2000, apoyadas por sus respectivos gobiernos y dirigidas a diversos ámbitos como el laboral, medioambiental, de transferencia tecnológica, competitividad, por citar solo algunos de ellos.
Durante el año 2000, el debate sobre la dimensión internacional que debía dársele a la RSE estaba centrado en la definición de normas de certificación de buenas prácticas de responsabilidad social empresarial, lo cual resultó ser de gran importancia para las empresas en cuanto a la preferencia de los stakeholders (consumidores, proveedores, accionistas, gobierno y comunidad), para con las empresas que demuestran tener incorporada la RSE entre sus prácticas empresariales, a lo que se suma la aplicación de políticas nacionales e internacionales de protección de los trabajadores y del medio ambiente.

Esta nueva estrategia en materia de RSE refleja el convencimiento de los estados miembros de la Unión Europea acerca del papel que la responsabilidad social empresarial puede desempeñar para alcanzar un crecimiento económico inclusivo, sostenible e inteligente.

Las autoras Chumaceiro y Hernández (2012) que consideran que es muy importante señalar todo lo relacionado con la actividad de Responsabilidad Social 
(en la práctica) debido a que tiempo atrás se creía que la política económica debía ser dejada a los gobiernos porque la empresa ya hacía "suficiente" referente a la sociedad, de manera que generaba riqueza y empleo. Ahora bien, siguen resaltando las autoras, no es secreto que el mundo empresarial está sometido a la regulación y planificación gubernamental.

De tal manera que para la adopción de los criterios de responsabilidad social (RS) en la gestión empresarial es medular la formalización de políticas y sistemas de gestión en los ámbitos económico, social y medioambiental; también, la transparencia informativa respecto de los resultados alcanzados en tales ámbitos; $y$, finalmente, el escrutinio externo de los mismos. Entonces, la RSE, es la forma en que las grandes empresas y corporaciones incorporan la ética en el plan de negocios en una perspectiva de largo plazo, para responder a las demandas de los distintos grupos de interés o stakeholders (incluyendo a los gobiernos) que tiene la empresa en la sociedad.

De igual manera las organizaciones no gubernamentales han estado experimentado un crecimiento en número de socios, además de haber ganado mayor confianza por parte de la sociedad civil y opinión pública en general. De acá que la RSE ha venido ganando importancia internacional, de ello dan cuenta los organismos internacionales como la Unión Europea (UE), la Organización para la Cooperación y el Desarrollo Económico $(\mathrm{OCDE})$, el Banco Mundial, entre otros, y la percepción de parte de los grandes inversionistas institucionales de que la RSE constituye un buen indicador acerca de la calidad en las prácticas de gestión y gobierno de las empresas.

Por su parte, las políticas públicas en torno a la responsabilidad social empresarial son promocionadas en su mayoría por procesos en Organismos internacionales, en tal sentido son varias las iniciativas, tanto públicas como privadas, que hacen referencia a la responsabilidad social y ambiental de las empresas.

Es por ello entonces que el presente artículo pretende reflejar el actual estado de los criterios de RSE, abordar la promoción de la RSE por parte de los organismos internacionales haciendo referencia a las principales iniciativas surgidas de estos y que en su mayoría se encuentran en el continente Europeo, fomentando la formalización de la adopción de sistemas de gestión, por parte de los gobiernos y empresas, en los tres pilares: económico, social, medioambiental, políticas orientadas al incremento de la transparencia de las compañías respecto a sus impactos económicos, sociales y ambientales. Políticas que incentivan un mayor escrutinio de los resultados RSE de las empresas.

\section{Metodología}

Hernández, Fernández y Baptista (2006:6) dicen que el "enfoque cualitativo utiliza recolección de datos sin medición numérica para descubrir - afinar preguntas de investigación y puede o no probar hipótesis en su proceso de interpretación".

Por su parte, el autor Tamayo y Tamayo (2009:57) argumenta que "la investigación cualitativa por su enfoque metodológico y su fundamentación epistemológica, tiende a ser de orden descriptivo, orientado a estructuras teóricas".

El presente estudio se encuentra enmarcado dentro del paradigma cualitativo, bajo un enfoque hermenéutico, su método será análisis de contenido. En tal sentido, Rodríguez, Gil Flores y García (1999) declaran lo difícil y polémico que resulta abreviar tanta diversidad en una tipología básica, sin embargo, siguiendo los aportes de Lincoln y Guba (1985), destacan una 
serie de niveles analíticos que permiten establecer unas características comunes de esta diversidad de enfoques y tendencias:

Nivel ontológico en el que se especifica forma y naturaleza de la realidad social y natural.

Nivel epistemológico, que parte de la realidad concreta y los datos que esta le aporta para llegar a una teorización posterior.

Nivel metodológico en el cual se sitúan los argumentos referidos a las formas de investigación en torno a la realidad.

Nivel técnico, la investigación se caracteriza por el uso de técnicas que permiten recabar datos que informen la particularidad de las situaciones, con ello se logra una descripción exhaustiva y densa de la realidad específica objeto de la investigación.

Nivel de contenido, la investigación cualitativa cruza todas las ciencias y disciplinas de tal forma que se desarrolla y aplica en política, educación, sociología, psicología, economía, medicina, antropología, etc.

En el desarrollo de la presente investigación se utilizó un enfoque cualitativo, pasando por todos sus niveles. Desde lo ontológico se analizó acerca del dinamismo y la interacción existente entre las categorías objeto de estudio. Epistemológicamente hablando se partió desde los datos bibliográficos reales y se realizaron análisis de los mismos.

El nivel metodológico se ha construido en la medida del avance del estudio. Para el nivel técnico se recolectó la información desde las diferentes fuentes, se seleccionaron los datos que pertenecían al conjunto de particularidades, explicitas en el estudio, lo que permitió una descripción exhaustiva de la realidad a estudiar. El contenido del presente estudio transversa varias disciplinas en las que destacan: sociedad, política, empresa y ambiente.

Por su parte, Zapata (2007) expresa que la sociedad posindustrial ha originado diversas escuelas y corrientes hermenéuticas que abordan descriptiva y comprensivamente la realidad. Es decir, que el enfoque hermenéutico busca interpretar y comprender los motivos internos de la acción humana, por medio de procesos libres y no estructurados, sino sistematizados, que tienen su fuente en la filosofía humanista, y que han facilitado el estudio de los hechos históricos, sociales, políticos y psicológicos del ser humano.

El análisis de contenido toma sus bases de la lingüística, particularmente de la semiótica, con la clara intención de interpretar el texto/acción. De manera tal, que en este estudio se utilizó la corriente hermenéutica mediante el análisis de contenido de los documentos pertinentes a las organizaciones internacionales que buscan promover la RSE en el mundo y el logro que las mismas han tenido en Europa.

Políticas para la promoción de RSE La RSE forma parte de las agendas de organismos internacionales y de ahí se traslada, con un enfoque temporal diverso, al diseño de programas públicos de carácter nacional. También es un hecho llamativo que se vaya constituyendo un "corpus doctrinal RSC", gracias a la confluencia de los diferentes modelos promovidos por las organizaciones internacionales. Así, con diversos enfoques, desde la década de 1980 y a lo largo de los años 90, se han presentado diferentes modelos RSE. (Chumaceiro, Hernández, Yory y Ziritt, 2013).

Durante 2001 y 2002 los foros y conferencias en los que se alude al papel gubernamental en el desarrollo del enfoque RSE se han multiplicado, en ellos aparece continuamente la discusión sobre voluntariedad u obligatoriedad en 
la adopción de políticas formales RSE. Independientemente de las opuestas posturas, se mantiene un consenso en cuanto a la labor de los gobiernos como facilitadores en la discusión y las consultas y creadores de redes de conocimiento, acordando en definitiva la necesidad de que los poderes públicos legitimen los estándares de RSE ya existentes.

De esta manera, existe un acuerdo en que los gobiernos, en primer lugar, han de facilitar el diálogo y el flujo de información entre los distintos actores implicados. Las administraciones cumplen este papel con su participación en las organizaciones internacionales y la posterior difusión de las iniciativas y compromisos formales, pero también a través de la promoción de las iniciativas de los foros multistakeholder y las de carácter normativo. Dentro de las políticas de promoción de RSE durante los años 2000, se pueden enumerar tres, que, a consideración de los investigadores son las más importantes:

a. Políticas públicas que fomentan la formalización de políticas y adopción de sistemas de gestión, por parte de las empresas en los tres pilares: económico, social y medioambiental: Estas son trasmitidas a través de organizaciones internacionales e iniciativas nacionales.

b. Políticas públicas orientadas al incremento de la transparencia de las compañías respecto a sus impactos económicos, sociales y medioambientales: supone para la empresa dar a conocer a los diferentes grupos de interés el impacto real de las políticas y sistemas de gestión sobre sus intereses. Entre las empresas que tienen mayor transparencia informativa están Royal Dutch Shell, Rio Tinto, BAA, ITT Flygt.

c. Políticas públicas que incentivan un mayor escrutinio de los resultados RSE de las empresas ha tenido su primera plasmación en los mercados financieros, aunque en los últimos tiempos también los mercados de producto comienzan a recompensar aquellas empresas con mejores prácticas acreditadas RSE, entre ellas están Nokia, Ericsson, First Group, Vodafone.

\section{Organizaciones internacionales que promueven la formalización} de la RSE. La revisión de literatura relacionada con el tema ha permitido identificar que las políticas que promueven la formalización de la RSE se han generado principalmente en escenarios internacionales. Al respecto el caso más conocido es el de la Unión Europea, la cual con la presentación del Libro verde en el 2001, se ha constituido en un referente para la definición de la RSE. Esta iniciativa está articulada con los lineamientos de la OCDE, el Pacto Global de las Naciones Unidas, el modelo del Global Reporting Initiative (GRI), entre otros.

Comisión Europea: Un antecedente clave en la tarea de promocionar las políticas públicas de RSE es, el libro verde, para fomentar un marco europeo sostenible para la responsabilidad social de las empresas que tenía por objetivo:

"Iniciar un amplio debate sobre cómo podría fomentar la Unión Europea la responsabilidad social de las empresas a nivel europeo e internacional, en particular sobre cómo aprovechar al máximo las experiencias existentes, fomentar el desarrollo de prácticas innovadoras, aumentar la transparencia e incrementar la fiabilidad de la evaluación y la validación. Propone... un enfoque basado en asociaciones más profundas en las que todos los agentes desempeñen un papel activo". (ESADE, 2002:6).

El Libro verde debía adquirir algún reflejo, a corto o mediano plazo, en las políticas nacionales de los países miembros. Este toma como punto de partida una definición de la RSE como voluntaria, un medio para que las empresas contribuyan 
a un cambio positivo para la sociedad y un medio ambiente más limpio, a su vez expresan esta responsabilidad ante los trabajadores y todos los demás grupos de interés de la empresa. (Chumaceiro, 2013)

La RSE se vincula con empresas exitosas, adoptando, pues, la filosofía de los stakeholder. Las dimensiones del enfoque RSE (a juicio de la Comisión Europea), pasan por la gestión de recursos humanos, la salud y seguridad laboral, la adaptación al cambio, la gestión de los impactos medioambientales, las relaciones con las comunidades locales, las políticas hacia socios, proveedores y consumidores, el respeto a los Derechos Humanos, se incluye también la extensión de esta responsabilidad social y medioambiental a toda la cadena de proveedores.

La iniciativa del Libro verde se enmarca en las líneas y directrices de la OCDE, el Global Compact de Naciones Unidas, y el modelo de comunicación de Global Reporting Initiative. La Comisión Europea colabora activamente en la revisión y difusión de las Líneas Directrices de la OCDE. Asimismo, Global Compact y Global Reporting Initiative anunciaron en el año 2002 un marco cooperativo, que formaliza la naturaleza complementaria y el objetivo común de ambas iniciativas. Se aprecia, por tanto, un proceso de coincidencias entre los modelos más relevantes.

Otro aspecto positivo de la Comisión Europea respecto al enfoque de RSE es, su naturaleza multistakeholder. Una de las características básicas de la RSE es el diálogo y consulta con los diferentes stakeholders, y la evasión de la toma de decisiones construida desde puntos de vista no contrastados. La postura de la Comisión Europea se ha convertido en referente para todo debate, conferencia o adopción de política formal en el tema de RSE.

Por otra parte, la Comisión Europea se encuentra orientada a promover el enfoque y facilitar el diálogo sobre la RSE que han consistido en la organización de conferencias y encuentros, como la celebrada en Bruselas en mayo de 2001 sobre las Mejores Prácticas Empresariales para la RSE, o la celebrada el mismo mes en Lisboa, la Primera Conferencia Europea sobre Triple Cuenta de Resultados (triple bottom line) en Europa.

Los países de la Unión Europea en sus mesas de trabajo, resumen sus principales temas, como: RSE y competitividad, contribución de la RSE al desarrollo sostenible, pymes, eficacia de los códigos de conducta, orientaciones para las prácticas de RSE, los etiquetados sociales (que el producto ha sido hecho completamente de un modo responsable tanto dentro como fuera del propio país) y las Inversiones socialmente responsables (ISR) adoptadas por los fondos de pensiones y los fondos de inversión individuales.

Organización Internacional del Trabajo (OIT): Fue en 1977 cuando la OIT dio a conocer la Declaración Tripartita de Principios sobre las empresas multinacionales y la política social. Los principales temas abordados en esta declaración cubren, resumidamente, temas tales como: empleo, promoción y oportunidades para los trabajadores de las empresas; características del trato laboral, capacitación y entrenamiento; políticas de salarios; edad mínima para trabajar; manejo de las relaciones laborales; mecanismo de consulta; solución de conflictos laborales; entre otras.

Esta declaración proporciona, además, una lista de los convenios y recomendaciones internacionales existentes sobre el trabajo e invita a los gobiernos de los Estados miembros de la OIT, a las organizaciones de trabajadores y empleadores, y a las empresas multinacionales que operan dentro de los territorios de los mismos 
a observar estos principios (Arroyo y Suárez, 2007). Por otro lado, la misma declaración ha servido de base para el estándar internacional SA8000 de certificación ética de normas laborales para empresas, de la organización Social Accountability International (SAI). (Chumaceiro, 2013).

\section{Organización para la cooperación y el desarrollo económico (OCDE): La OCDE agrupa a las principales economías desarrolladas en el mundo y a otras en vías de desarrollo, además de miembros que son socios, pero no miembros plenos, como Chile. Uno de los elementos que más ha aportado desde este organismo para la aplicación de normas de responsabilidad social en las grandes empresas lo constituye el documento intitulado: Líneas directrices de la OCDE para empresas multinacionales, elaborado en 2000.}

Las directrices son según Chumaceiro (2013) de adhesión voluntaria, pero aquellas empresas que las adopten se comprometen a aplicar sus principios en todos los países en los que operan. Estas directrices son principios y normas voluntarias compatibles con la legislación en los países donde se aplican. Buscan la armonización con las políticas públicas locales, promueven la confianza entre las empresas y las sociedades, buscan potenciar un clima para la inversión extranjera y contribuir al desarrollo sostenible.

Las líneas incluyen los elementos habituales de la definición de responsabilidad social empresarial: desarrollo sostenible, respeto a los derechos humanos, colaboración con la comunidad, formación de los trabajadores, salud y seguridad laboral, buen gobierno corporativo, sistemas de gestión, difusión y promoción de las políticas corporativas, extensión de las exigencias propias a proveedores y subcontratistas, etc. A lo largo de los distintos capítulos se ofrecen recomendaciones, que deberían guiar las prácticas de las empresas en los distintos campos, y comentarios explicativos sobre su aplicación.

También se convoca a las empresas a la transparencia a través de la publicación de resultados financieros, objetivos $y$ declaraciones de valores, principales accionistas, miembros del consejo de administración, salarios de los altos directivos, y otros indicadores de los habitualmente usados para evaluar el buen gobierno. En este sentido, las recomendaciones sobre reporting, son mucho menos ambiciosas que las contenidas en el modelo de Global Reporting Initiative.

Las líneas directrices de la OCDE para empresas multinacionales son especialmente relevantes en cuanto a su potencial para la implicación gubernamental en la promoción de la RSE, en tanto que los gobiernos de la OCDE tienen como misión establecer Puntos Nacionales de Contacto encargados de su difusión.

The Global Compact. Pacto Mundial de las Naciones Unidas para la RSE: Global Compact o Pacto Mundial es una iniciativa que promovida por Kofi Anan desde Naciones Unidas en 2000. Insta a los líderes empresariales y las corporaciones para que, voluntariamente, trabajen en conjunto con las agencias de Naciones Unidas, trabajadores y la sociedad civil en apoyar principios ambientales y sociales de carácter universal.

\section{Global Compact, promueve 10 principios en cuatro ámbitos:}

Derechos Humanos: (1) Las compañías deben apoyar y respetar la protección de los derechos humanos proclamados a nivel internacional y (2) evitar verse involucradas en abusos de los derechos humanos.

Normas laborales: (3) Las empresas deben respetar la libertad de asociación 
y el reconocimiento efectivo del derecho a la negociación colectiva, (4) la eliminación de todas las formas de trabajo forzoso y obligatorio; (5) la abolición efectiva del trabajo infantil y (6) la eliminación de la discriminación respecto del empleo y la ocupación.

Medioambiente: (7) Las empresas deben apoyar la aplicación de un criterio de precaución respecto de los problemas ambientales, (8) adoptar iniciativas para promover una mayor responsabilidad ambiental y (9) alentar el desarrollo y la difusión de tecnología inocuas para el medioambiente.

Anticorrupción: (10) Las empresas deben trabajar contra la corrupción en todas sus formas, incluidas extorsión y soborno.

El programa establece la colaboración entre las empresas y las diferentes organizaciones de Naciones Unidas, las organizaciones empresariales, las instituciones que trabajan en el campo de la RSE, los sindicatos, las ONG para construir redes y proyectos conjuntos en los que se compartan los valores y los principios de RSE (los Derechos Humanos, los derechos laborales y el medio ambiente).

El programa no establece un cuerpo normativo internacional sino que es un programa voluntario, de carácter no vinculante, sin que implique ninguna obligación jurídica al respecto. No obstante, el programa adquiere importancia por el hecho de que está impulsado por el propio secretario general de Naciones Unidas y por el apoyo recibido por parte de grandes empresas en todo el mundo.

\section{Corporación Financiera}

Internacional - Equator Principles:

Los Equator Principles, o principios de igualación, están orientados a las entidades bancarias y son normas de adhesión voluntaria impulsadas por la Corporación Financiera Internacional
(IFC, sus siglas en inglés), agencia dependiente del Banco Mundial y fueron presentadas en 2003. Nacieron en 2002 a partir de una reunión de bancos organizada por la IFC para discutir los temas ambientales y sociales en los proyectos de inversión. Los bancos acordaron desarrollar un criterio común para evaluar el riesgo en estos temas, que condujo posteriormente a los 17 principios.

La adhesión a los principios implica que los bancos, además de la evaluación crediticia para el financiamiento de los grandes proyectos de inversión, sumen criterios sociales y ambientales. Una vez concedidos los recursos, se lleva a cabo un seguimiento para asegurarse de que las empresas beneficiarias de crédito cumplan estos estándares.

Los aspectos según Chumaceiro (2013), considerados por los principios son: desarrollo sostenible y uso de recursos naturales renovables; protección de la salud, diversidad cultural y biodiversidad (inclusión de especies en peligro y ecosistemas sensibles); sistemas de salud y seguridad (prevención de incendios), impactos socioeconómicos, sobre personas y comunidades indígenas y sobre proyectos anteriores o futuros; sistemas de adquisición y uso del suelo; reasentamientos involuntarios de población; participación de sectores afectados en el diseño e implementación del proyecto; consideración de alternativas preferibles en términos sociales y ambientales; eficiencia en la producción, transporte y consumo de energía, y prevención y control de la contaminación y optimización de la gestión de residuos.

Losprincipiospermitenquelascompañías bancarias puedan categorizar el riesgo ambiental y social. Si no cumplen con los principios, se incrementa la tasa de riesgo $y$, con ello, se encarece el crédito. Los principios se aplican a proyectos cuyo costo es de USMM $\$ 50$ o más, e incluye el impacto ambiental 
sobre flora y la fauna, la exigencia de compensaciones monetarias para las poblaciones afectadas por la iniciativa, la protección a comunidades indígenas y la prohibición de financiar el uso de trabajo infantil o esclavo.

Los estándares de los proyectos sociales y medioambientales a considerar son: (1) Clasificación de los proyectos según criterios de impacto social $y$ medioambiental en tres categorías, $A$ : riesgo alto, B: riesgo medio $\mathrm{y}, \mathrm{C}$ : riesgo bajo, (2) evaluación medioambiental y social de proyectos que han sido clasificados como A o B, (3) formulación de un plan de gestión medioambiental para todos los proyectos A y para aquellos proyectos $B$ que el banco estime. Dicho plan deberá proponer planes de mitigación, seguimiento y gestión de riesgos sociales y medioambientales para reducir los riesgos de la comunidad donde van a ser implementados, y, (4) los contratos de financiamiento deberán incluir cláusulas de cumplimiento con los estándares sociales y medioambientales y el Plan de Gestión Medioambiental.

Banco Mundial:

El Banco Mundial mantiene un programa sobre Responsabilidad Social Empresarial y Competitividad Sostenible que, al igual que el Pacto Mundial de Naciones Unidas, fue presentado en la ocasión del Foro Económico Mundial de Davos, en enero de 2000. El plan del Banco Mundial está incluido en un programa más amplio sobre Gobierno Corporativo y Competitividad, que a su vez se encuentra enmarcado en la estrategia de reducción de la pobreza.

La táctica del Banco Mundial sobre RSE, es que cuenta con socios como el Programa de Naciones Unidas para el Desarrollo, la OCDE, y Harvard Business School. Esta táctica se centra primeramente en la investigación y en ofrecer recursos y formación, dirigida a gestores, escuelas de negocios, periodistas, sector no gubernamental y sector público de aquellos países en vías de desarrollo. La formación incluye cursos en diferentes países, conferencias mediante listas de correos en Internet que cuentan con una amplia participación, y seminarios que se llevan a cabo a través de la red de instituciones asociadas $\mathrm{y} / \mathrm{o}$ dependientes del Banco Mundial.

Durante el año 2002 el Banco Mundial llevó a cabo una encuesta mundial sobre la RSE que sirvió para orientar futuras actividades de formación y divulgación hacia el fortalecimiento de los conocimientos gerenciales sobre buenas prácticas de RSE, también para incrementar las competencias de altos funcionarios gubernamentales en el enfoque de RSE, y a promover los incentivos económicos a las mejores prácticas empresariales.

Una parteconsiderable de la participación en el programa sobre RSE del Banco Mundial proviene de países del este de Europa y de la antigua Unión Soviética, ámbito geográfico al que más recursos ha dedicado esta institución para la promoción y el fortalecimiento de buenas prácticas de gobierno corporativo. En tal sentido, el Banco Mundial, la OCDE, el Centro para la Empresa Privada Internacional y la Asociación para la Protección del Inversor han puesto en marcha la Russian Corporate Governance Roundtable, mesa que sirve de plataforma para otras actividades RSE del Banco Mundial en este país.

\section{Organizaciones internacionales que incentivan la transparencia de la}

RSE. Actualmente el posicionamiento de las empresas en los mercados nacionales e internacionales depende no solo de su competitividad, sino de la credibilidad y reputación que han logrado consolidar con sus stakeholders. En este orden de ideas, una empresa socialmente responsable no puede basar su éxito en decisiones de eficiencia económica, debe incluir aspectos como la transparencia con sus grupos de interés, dado que está condicionada a contribuir a la construcción de una 
cultura socialmente responsable que le permita diferenciarse en el mercado de aquellas empresas que se consideran socialmente responsables porque mitigan sus externalidades o porque pueden ejercer acciones filantrópicas.

Comisión Europea: La Comisión Europea en el Libro verde y en las posteriores comunicaciones ha evidenciado la necesidad de información y transparencia en su proceso de promoción de la RSE, especialmente por la adopción del modelo de Global Reporting Initiative (GRI) como base a sus propuestas. Sin embargo, en el Foro Europeo Multi-stakeholder para la RSE (EMS CSR Forum) no participa el GRI como tal, pese a haber establecido su sede en Amsterdam.

En general según Lafuente, Viñuales, Pueyo y Llaría (2003) la Comisión Europea es reservada en cuanto a la posibilidad de crear una directiva sobre transparencia y comunicación de RSE, pedida por Francia y otros gobiernos de la UE. En tal aspecto Lafuente, Viñuales, Pueyo y Llaría, (2003:46) exponen que: "Desde Bruselas se desea abandonar los estándares contables regionales en favor de estándares internacionales e incluir una perspectiva de triple cuenta de resultados, aunque la conversión de estas intenciones en requerimientos se deja para el largo plazo. De todas maneras, la Comisión recomienda la inclusión, cuantitativa, de un balance social y medioambiental en los informes anuales".

\section{Global Reporting Initiative: El} modelo tiene un origen multidisciplinar y multi-stakeholder, lo cual es una fortaleza para el mismo, ya que para su elaboración colaboran un conjunto de empresas, las Naciones Unidas y organizaciones académicas como Harvard University. El modelo desarrolla indicadores para el reporte corporativo en materia social y medioambiental. Facilita a las empresas identificar qué es relevante comunicar. Así en el GRI se pueden encontrar indicadores para medir la relación con empleados, la gestión de la cadena de proveedores o la gestión del medio ambiente, lo cual ofrece un marco referencial que permite a las compañías identificar y organizar sus propios indicadores más relevantes en los ámbitos económico, social y medioambiental.

Global Reporting Initiative ofrece un marco adecuado para el cambio hacia la sostenibilidad empresarial, al obligar a las empresas a adecuar sus sistemas de gestión a los objetivos de comunicación que se han marcado y facilitar la comparación y la verificación de los resultados empresariales.

Organizaciones internacionales
que impulsan el escrutinio de la RSE. A continuación se destacan los siguientes organismos internacionales que estimulan el escrutinio de la RSE de los gobiernos y las empresas.

\section{Comisión Europea:}

La Comisión Europea ha organizado foros, encuentros y grupos de trabajo dedicados a analizar la posible convergencia y los modos de verificación de los códigos de conducta y su relación con los sellos sociales, desde mediados de la década del 2000. En este sentido, en el Libro verde se plantea promover un marco europeo para la RSE, las "iniciativas de etiquetado social y ecológico tienen un alcance y un impacto potencial limitados, porque se circunscriben a nichos específicos del mercado de la venta al por menor... lo que indica que debe mejorarse la eficacia de dichas etiquetas" (ESADE, 2002:35).

En tal sentido, Zeilinger, (2001), expresa que a menudo se ha mencionado el absurdo de que los consumidores que deciden aplicar criterios RSE en sus compras puedan verse obligados a escoger entre, por ejemplo, "café laboralmente responsable" o "café medioambientalmente responsable", 
y se ha solicitado a los gobiernos que legitimen iniciativas que unifiquen ambos criterios.

Por su parte, el informe final del Foro Europeo Multistakeholder menciona a la inversión con criterios de responsabilidad social empresarial, inversión socialmente responsable, al consumo socialmente responsable, y a la contratación pública con criterios de RSE como los principales impulsores externos de la misma.

Norma SA8000: SA8000 es una norma auditable elaborada por Social Accountability International (SAI), una organización no gubernamental fundada en 1997. El punto de partida del desarrollo de la norma son los principios de la Organización Internacional de Estandarización (ISO), en el cual han participado empresas y organizaciones de la sociedad civil, además de otras iniciativas empresariales que cuentan con apoyo gubernamental, como es el caso del Apparel Industry Partnership/ Fair Labour Agreement de EEUU.

La norma abarca los principales acuerdos internacionales sobre derechos laborales, la Declaración Universal de Derechos Humanos, y la Convención de Naciones Unidas sobre los Derechos del Niño. En octubre de 2002, 172 empresas en 127 países contaban con una acreditación sobre la norma SA8000, tres de ellas españolas: Dole Pascual, Ramel S.A. y Fitman S.A. resaltando que el país que cuenta con un número mayor de empresas certificadas es China, la mayor parte manufactureras.

Esta iniciativa podría clasificarse también como promotora y fortalecedora de políticas formales RSE debido a que buena parte de sus contenidos son asimilables a los de un sistema de gestión tradicional, pero el hecho de que ofrezca los medios y requiera ser verificada, la convierte en un destacado instrumento de verificación de RSE.
La norma SA8000 ofrece elementos y procesos necesarios para la auditoría social, a través de peritos especializados, autorizados y formados por la propia SAI.

SA8000, aunque algunas agencias gubernamentales de USA figuran en su Consejo Asesor, no es una iniciativa gubernamental. Sin embargo, diversos gobiernos han comenzado a valerse de esta norma, sea exigiendo criterios similares para la obtención de sellos sociales, haciendo que la norma sea adoptada por las agencias gubernamentales para satisfacer determinadas exigencias de consumidores en sus respectivos países.

\section{Conclusiones}

El objetivo es extender la cultura de la RSE al conjunto de la sociedad a través de actuaciones como la promoción de sus principios, de las recomendaciones de consenso internacional en esta materia y la difusión de buenas prácticas en este ámbito.

La RSE es un elemento impulsor de la sostenibilidad, de la igualdad de oportunidades y de la cohesión social. Este aspecto es importante especialmente en organizaciones como: Empresas con actividad desarrollada en mercados exteriores y/o que dependan de dichos mercados.

Pequeñas y medianas empresas.

Administraciones públicas, incluyendo el sector público empresarial.

Empresas de la economía social.

La RSE y su razón pueden ser concebidos como un instrumento estratégico para lograr ventajas competitivas. Es posible entenderla también desde los puntos de vista normativo, ético, moral y/o social. Estos últimos argumentos sociales, públicos y legales son los que defienden las organizaciones sociales al hablar de la RSE. Se ha analizado el papel activo 
de las organizaciones sociales como generador de RSE y su papel clave dentro del enfoque de autorregulación que domina el espacio de la RSE en la actualidad, también cumplen un papel fundamental en la labor de divulgación, difusión y sensibilización en RSE fundamental para el avance de la misma y en la concienciación ciudadana.

Ahora bien los elementos generales que abordan las propuestas y políticas públicas de RSE se pueden abordar en el contexto de la legislación internacional y la adhesión a pactos internacionales, entendiendo que:

En el primer caso, en el contexto europeo y de los países de OCDE hay líneas directrices cuyos países miembros y socios han de observar para el desarrollo de actividades de negocios responsables. Las hay para las actividades de las empresas multinacionales y para la formación de los gobiernos corporativos.

Las industrias multinacionales tienen cuerpos específicos, y las más exigentes se encuentran en los sectores que contaminan el medioambiente. Cuando los países no cuentan con legislación propia han de recurrir a estas prácticas globalmente aceptadas, y, también, definir políticamente cuánto están los países dispuestos a contaminar.

En el entendido de los países que no cuentan con legislación propia es importante destacar que, el Pacto Global de Naciones Unidas une criterios de comportamiento de fácil adsorción para las empresas, tanto en los impactos directos en los ciudadanos como en ámbitos de la competencia desleal, la extorsión y el soborno.

\section{Referencias}

Arroyo, Gonzalo y Suárez, Andrés. (2006). Responsabilidad social corporativa. Una mirada global. Chile: Universidad Alberto Hurtado.

Chumaceiro Ana, Hernández de Velazco, Judith, Yori Ligia y Ziritt, Gertrudis. (2013), Responsabilidad social empresarial y políticas pública. Revista de ciencias sociales (Ve), vol. XIX, núm. 2, abril-junio, 2013, pp. 309-321. Universidad del Zulia. Maracaibo, Venezuela.

Chumaceiro Ana.; Hernández de Velazco, Judith. (2012) La legislación tributaria venezolana como promotora de la responsabilidad social empresarial. Revista OIKOS. Vol 16, No 33. Universidad Católica Silva Henrique. Chile.
Chumaceiro, Ana, (2013). Políticas internacionales, nacionales y políticas públicas tributarias en Venezuela como promotoras de la responsabilidad social de las empresas. Tesis Doctoral. (Doctorado en Ciencias Políticas) Universidad Dr. Rafael Belloso Chacín. Maracaibo.

Comisión Europea (2004): Corporate social responsibility, national public policies in the European Union, (Luxemburgo, Office for Official Publications of the European Communities).

Comisión Europea. (2001). Libro verde: fomentar un marco europeo para la responsabilidad social de las empresas. Disponible en línea http:// thegreenbook.eu/ellibroverde.pdf (Consultado el 14/02/2015) 
ESADE, (2002). Libro verdede la comisión europea: Fomentar un marco europeo para la responsabilidad social de las empresas. Comunicación de la comisión relativa a la responsabilidad social de las empresas: una contribución empresarial al desarrollo sostenible Barcelona: IPES.

Hernández Sampieri, Roberto; Fernández Collado, Carlos; Baptista Lucio, Pilar. (2006) Metodología de la investigación. México. Mc Graw Hill.

Lafuente, Alberto, Viñuales, Victor, Pueyo, Ramón y Llaría, Jesús. (2003). Responsabilidad social corporativa y políticas públicas. Madrid: Fundación Alternativas.

Lincoln, Yvonna. y Guba, Egon. (1985). Naturalisíic inquirí. Beverly Hills: Sage Publications.

Organización para la Cooperación y el Desarrollo Económico (1999): OCDE Guidelines for multinational enterprises.Paris: OCDE.

Organización para la Cooperación y el Desarrollo Económico (2001a): Corporate Social Responsibility. Partners for Progress. Paris: OCDE.
Organización para la Cooperación y el Desarrollo Económico (2001b): The OECD Guidelines for multinational enterprises: Annual Report 2001. París: OCDE.

Organización para la Cooperación y el Desarrollo Económico (2003): Annual Report on the OCDE Guidelines for Multinational Enterprises: 2003 Edition. París, OCDE.

Organización para la Cooperación y el Desarrollo Económico (2004): Implementing sustainable development: Key Results 20012004. París, OCDE.

Rodríguez, Gregorio, Gil Flores, Javier y García, Eduardo. (1999). Metodología de la investigación cualitativa. Málaga: Aljibe

Tamayo y Tamayo, Mario. (2009). Técnicas de investigación. Editorial Me Graw Hill. México.

Zapata, R. (2007). "Olas paradigmáticas de las estrategia: enfoque hermenéutico". Revista Ciencias Estratégicas. Vol 15. N¹7. p 83-92.

Zeilinger, I., (2001). Labour standards and government programs. Amsterdam, Royal Tropical Institute. 